Relations industrielles

Industrial Relations

\title{
Industrial Relations Handbook; Ministry of Labour, London, Her Majesty Stationery Office, 1961. 234 pp.
}

Jean-Réal Cardin

Volume 17, numéro 1, janvier 1962

URI : https://id.erudit.org/iderudit/1021670ar

DOI : https://doi.org/10.7202/1021670ar

Aller au sommaire du numéro

Éditeur(s)

Département des relations industrielles de l’Université Laval

\section{ISSN}

0034-379X (imprimé)

1703-8138 (numérique)

Découvrir la revue

Citer ce compte rendu

Cardin, J.-R. (1962). Compte rendu de [Industrial Relations Handbook; Ministry of Labour, London, Her Majesty Stationery Office, 1961. 234 pp.] Relations industrielles / Industrial Relations, 17(1), 97-98.

https://doi.org/10.7202/1021670ar

Tous droits réservés @ Département des relations industrielles de l’Université Laval, 1962
Ce document est protégé par la loi sur le droit d'auteur. L'utilisation des services d'Érudit (y compris la reproduction) est assujettie à sa politique d'utilisation que vous pouvez consulter en ligne.

https://apropos.erudit.org/fr/usagers/politique-dutilisation/ 
Psychologie sociale de lindustrie, par J.A.C. Brown. Traduction de M. et Y. Brès; préface de Juliette FavezBoutonier. Editions de l'Epi, 13, rue Séguier, Paris, 6. 1961. 352 pages.

Les familiers de la littérature en relations industrielles de langue anglaise connaissent déjà \& The Social Psychology of Industry » paru en Grande-Bretagne en 1954. Ils savent la valeur de cet ouvrage remarquable écrit par un maître de la psychosociologie industrielle.

Nous sommes heureux qu'un éditeur ait accepté d'en faire faire la traduction on langue française.

Enfin, les spécialistes en relations industrielles, directeurs d'usine, directeurs du personnel, techniciens, dirigeants ouvriers, tout comme les profanes auront à leur disposition une étude de synthèse qui leur permettra de comprendre dans leur langue le problème des relations humaines dans l'entreprise et de l'organisation sociale de la communauté de travail.

L'ouvrage du Dr Brown, qui est déjà considéré comme un classique, connaìtra sans doute une large diffusion dans les milieux intéressés aux problèmes de relations industrielles au Canada français. Il le mérite.

\section{GÉrard Dion}

Industrial Relations Handbook; Ministry of Labour, London, Her Majesty Stationery Office, 1961. 234 pp.

Voici un petit livre qui tend à renseigner l'observateur étranger sur le système britannique des relations industrielles. C'est une publication du Ministère du Travail de Grande-Bretagne, publication qui paraît déjà depuis 1944 . Il s'agit ici de la dernière édition alors qu'une seconde en avait été faite en 1953.

Comme on le dit en préface, cet ouvrage n'avait pas originellement été écrit pour fins de publication mais pour l'usage interne du personnel du Ministère du Travail anglais. Mais, comme il arrive quelquefois, lorsque le sujet traité est d'intérêt général et que la qualité de sa présentation impose l'ouvrage à un public plus vaste, la demande pour ce Handbook, aussi bien en Grande-Bretagne que dans le reste des pays du Com- monwealth et au-delà, a fait qu'au-delà de cent mille copies en ont été vendues depuis la date de sa première parution.

Cet ouvrage présente d'une façon succincte mais très bien ordonnée, sans commentaires autres que ceux techniquement nécessaires à l'intelligence du sujet traité ni jugements de valeur, les faits essentiels concernant les organisations d'employeurs et de travailleurs en Grande-Bretagne; il donne une description sommaire des procédés de négociations collectives dans les différents secteurs de l'économie britannique, du contenu de ces négociations ainsi que des procédures de règlement des conflits ou griefs qui surgissent à l'occasion des relations collectives de travail. Il décrit aussi, historiquement et analytiquement les politiques de relations de travail issues, soit des lois statutaires, soit des coutumes ou ententes volontaires entre les parties en présence.

Quant on connait le caractère extrèmement diversifié des organisations syndicales et des procédés en relations industrielles en Angleterre, ainsi que l'absence de lois - cadres d'application générale et contraignante, comme c'est le cas au Canada et aux Etats-Unis, avec nos lois de relations ouvrières; si l'on songe surtout que presque toutes les relations ouvrières patronales du Royaume-Uni, se sont développées sur la base, très respectée là-bas, du 《volontariat 》 et que seuls quelques grands textes de principes en matière juridique et administrative ont guidé ces développements, on apprécie davantage l'effort de systématisation dont fait preuve ce petit livre et le caractère ordonné qu'il donne à la présentation d'une telle situation. C'est essentiellement là que réside son mérite: il permet au lecteur, même non averti, de se retrouver dans ce que les Webbs ont déjà appelé « un chaos d'atomes ».

Le livre se divise en trois parties. La première, qui occupe le plus d'espace et revêt une importance particulière, est surtout consacrée à rendre compte des procédés et des formes d'organisation des employés et des employeurs. Elle débute par l'exposé des principaux alons historiques survenus depuis les cent-cinquante dernières années et indique les principaux textes législatifs qui ont accompagné ou reflété ces différentes étapes. 
Etant donné que la caractéristique essentielle de l'évolution des relations industrielles en Angleterre a été l'organisation volontaire de ces relations, le manuel insiste davantage sur la présentation des procédés de négociations conjointes entre employeurs et syndicats. Le chapitre II, fait état du développement de la convention collective et indique ses principales méthodes. Les trois chapitres suivants (Ch. III, IV, V), décrivent avec assez de précision les formes que revêt la négociation conjointe respectivement dans le secteur privé, les industries nationalisées et les services publics. Le chapitre VI, est consacré plus précisément à la description des rouąges de représentation et de négociation à l'échelle de l'usine ou du lien du travail.
La seconde partie rend compte des voies selon lesquelles le Ministre du Travail intervient dans les relations industrielles et présente les textes statutaires qui servent de base à son action en ce domaine. Il traite de la conciliation, des formes d'arbitrage volontaire auxquelles les parties peuvent recourir en cas de différends; les lois de taux minimums de salaires jouant en certains secteurs, etc.

La troisième partie fournit certains renseignements au sujet des salaires, des heures de travail, des congés statutaires, etc., en un mot, de ce qui fait généralement l'objet de la convention collective.

\section{Jean-Réal, Cardin}

\section{PUBLICATIONS RÉCENTES RECENT PUBLICATIONS}

\section{Généralités}

Canadian Labour, Vol. 7, No. 1, January 1962: \& Labour's Concern for Human Rights, by Frank Hall, pp. 5-7: « Ontario's Program for Human Rights, by Tom Eberlee, pp. 8-10: \& Fair Practice Laws - the Canadian Experience, by Sid Blum, pp. 11-13.

Enquêtes sur les conditions de vie des familles - Recueil de monographies, par Bureau international du travail, Genève, $1961,330 \mathrm{pp}$.

Développement et Civilisations, no 8, octobre-décembre 1961: "Plannification et Développement », par Gabriel Turin, pp. 55-65: \& La Coopération à travers le monde \$, pp. 73-78.

«Démocratie et développements 》, par Louis-Joseph Lebret, Développement et Civilisations, no 7, juillet-septembre 1961.

《 La region economica $\gg$, par Jacques $R$. Boudeville (Paris), Economica, vol. V, nos 17-20, juillet 1958 - janvier 1959, pp. 51-157,
* Economic and Technology: The Case of Soviet Steel », by M. Gardner Clark, New York State School of Industrial and Labor Relations, Reprint No. 109.

«Travail. fatigue et équilibre mental », Revue de l'Action Populaire, no 15.3, décembre 1961, pp. 1171-1190.

«L'Eglise et le problème social », Labor. no 5, 34ème Année, 1961, pp. 194-197.

«Quelques réflexions sur l'essor de la Recherche Opérationnelle», par Robert Faure, Paris, Management International, no 3, mai-juin 1961, pp. 46-50.

«L'Administration des Entreprises en tant que discipline scientilque », par Dott. Enrico de Gennaro, Torino, Management International, no 3 , mai-juin 1961, pp. 90-95.

«L'Encyclique Mater et Magistra », par le R.P. Joseph Joblin, S.J., Revue Internationale du Travail, vol. LXXXIV, no 3 , septembre 1961, pp. 139-154.

« La quarante-cinquième session de la Conférence internationale du Travail, 